\title{
OPERATORS WITH FINITE CHAIN LENGTH AND THE ERGODIC THEOREM
}

\author{
K. B. LAURSEN AND M. MBEKHTA
}

(Communicated by Palle E. T. Jorgensen)

\begin{abstract}
With a technical assumption $(E-k)$, which is a relaxed version of the condition $T^{n} / n \rightarrow 0, n \rightarrow \infty$, where $T$ is a bounded linear operator on a Banach space, we prove a generalized uniform ergodic theorem which shows, inter alias, the equivalence of the finite chain length condition $(X=$ $\left.(I-T)^{k} X \oplus \operatorname{ker}(I-T)^{k}\right)$, of closedness of $(I-T)^{k} X$, and of quasi-Fredholmness of $I-T$. One consequence, still assuming $(E-k)$, is that $I-T$ is semi-Fredholm if and only if $I-T$ is Riesz-Schauder. Other consequences are: a uniform ergodic theorem and conditions for ergodicity for certain classes of multipliers on commutative semisimple Banach algebras.
\end{abstract}

\section{OPERATORS WITH FINITE CHAINS}

We begin with a few algebraic observations. Let $S$ be a linear operator on the vector space $X$. If there is an integer $n$ for which $S^{n} X=S^{n+1} X$, then we say that $S$ has finite descent and the smallest integer $d(S)$ for which this equality occurs is called the descent of $S$. If there is an integer $m$ for which $\operatorname{ker} S^{m}=\operatorname{ker} S^{m+1}$, then $S$ is said to have finite ascent and the smallest integer $a(S)$ for which this equality occurs is called the ascent of $S$. If both $a(S)$ and $d(S)$ are finite, then they are equal [3], 38.3; we say that $S$ is chain-finite and that its chain length is this common minimal value. Moreover ([3], 38.4), in this case there is a decomposition of the vector space

$$
X=S^{d(S)} X \bigoplus \operatorname{ker} S^{d(S)} .
$$

Thus in particular, if $S$ has chain length at most 1 , then $X=S X \bigoplus \operatorname{ker} S$. There is a way of describing this. By a complement of a subspace $Y \subseteq X$ we mean a subspace $Z$ of $X$ for which $X=Y \oplus Z$, as an algebraic direct sum.

Proposition 1. If $S$ is a linear operator on a vector space $X$, then $S$ has chain length at most 1 if and only if $S$ has finite descent and $\operatorname{ker} S$ has an $S$-invariant complement.

Proof. It is clear from the above remarks that the condition of this proposition is necessary for finite chain length. On the other hand, if $\operatorname{ker} S$ has an $S$-invariant complement $Z$, then $X=Z \bigoplus \operatorname{ker} S$ and $S Z \subseteq Z$. Thus $S X \cap \operatorname{ker} S=$

Received by the editors May 2, 1994.

1991 Mathematics Subject Classification. Primary 47A10, 47A35.

(c) 1995 American Mathematical Society 
$S Z \cap \operatorname{ker} S=\{0\}$, and hence $\operatorname{ker} S^{2}=\operatorname{ker} S$, so that $S$ has ascent 0 or 1 . Consequently, since $S$ has finite descent, $S$ is chain-finite with chain length at most 1 , by [3], 38.3.

We now focus on the topological situation: assume that $X$ is a Banach space and fix an element $T \in L(X)$, where $L(X)$ denotes the Banach algebra of continuous linear operators on $X$. We are interested in the behavior of the averages

$$
M_{n}(T):=\left(I+T+T^{2}+\cdots+T^{n-1}\right) / n
$$

as $n \rightarrow \infty$. For reasons that will become clearer later we shall make the following assumption on $T$.

Definition 2. Let $k \in \mathbf{N}$ and $T \in L(X)$. We say that $T$ satisfies condition $(E-k)$ if $\left\|(I-T)^{k} M_{n}(T)\right\| \rightarrow 0$ as $n \rightarrow \infty$.

It is clear that condition $(E-k)$ implies condition $(E-n)$ for any $n \geq k$.

Lemma 3. $T \in L(X)$ satisfies condition $(E-k)$ if and only if $(I-T)^{k-1} T^{n} / n \rightarrow$ 0 as $n \rightarrow \infty$.

Proof. An easy induction shows that $(I-T)^{k} M_{n}(T)=-(I-T)^{k-1} T^{n} / n$ for all $k, n \in \mathbf{N}$.

Thus we see that $(E-1)$ is equivalent to the condition $\left\|T^{n} / n\right\| \rightarrow 0$ as $n \rightarrow \infty$ and that $(E-2)$ is the same as the condition $\left\|\left(T^{n}-T^{n+1}\right) / n\right\| \rightarrow 0$ as $n \rightarrow \infty$. The example $T:=\left[\begin{array}{ll}1 & 1 \\ 0 & 1\end{array}\right]$ shows that $(E-2)$ is strictly weaker than $(E-1)$. Note also that Lemma 3 shows that condition $(E-k)$ is the same as condition $(E-1)$ for the restriction $T \mid\left((I-T)^{k-1} X\right)^{-}$.

There is a simple but significant connection between the concepts mentioned so far.

Proposition 4. Suppose $T \in L(X)$ satisfies condition $(E-k)$. Then $a(I-T) \leq k$. Proof. Let $x \in \operatorname{ker}(I-T)^{k+1}$. Put $w:=(I-T)^{k-1} x$ and $y:=(I-T) w$. Then $y \in(I-T) X \cap \operatorname{ker}(I-T)$, in particular $T y=y$. We obtain $T w=w-y$, hence, for every $n \geq 1, T^{n} w=w-n y$. Thus $y=\left(I-T^{n}\right) w / n=\left(I-T^{n}\right)$. $(I-T)^{k-1} x / n=(I-T)^{k-1} x / n-T^{n}(I-T)^{k-1} x / n$. If $n \rightarrow \infty$, then obviously $(I-T)^{k} x / n \rightarrow 0$, and since, by Lemma $3, T^{n}(I-T)^{k-1} x / n \rightarrow 0$, we conclude that $y=0$, and hence $x \in \operatorname{ker}(I-T)^{k}$.

Definition 5. An operator $S \in L(X)$ is said to be a quasi-Fredholm operator if it satisfies the following conditions: There are closed $S$-invariant subspaces $M$ and $N$ such that $X=M \bigoplus N$ and such that $S \mid N$ is nilpotent, while $(S \mid M)(M)$ is closed and contains all kernels $\operatorname{ker}(S \mid M)^{n}, n \in \mathbf{N}$.

An important result of Kato [4], Theorem 4, shows that this class of operators contains all semi-Fredholm operators. In the Hilbert space case, the quasiFredholm operators were introduced by Labrousse [5] and studied there and also in [8]; closed, densely defined quasi-Fredholm operators on Banach spaces have been considered in [10].

Theorem 6. If $T \in L(X)$ satisfies condition $(E-k)$, then the following statements are equivalent.

(a) I - T has chain length at most $k$. 
(b) 1 is a pole of the resolvent of order at most $k$.

(c) $I-T$ is a quasi-Fredholm operator.

(d) $(I-T)^{k} X$ is closed and $\operatorname{ker}(I-T)^{k}$ has a closed $T$-invariant complement.

(e) $(I-T)^{k} X+\operatorname{ker}(I-T)^{k}=(I-T)^{k} X \bigoplus \operatorname{ker}(I-T)^{k}$ is closed.

(f) $(I-T)^{m} X$ is closed for all $m \geq k$.

(g) $(I-T)^{k} X$ is closed.

(h) $(I-T)^{m} X$ is closed for some $m \geq k$.

(i) $I-T$ has finite descent.

Proof. The equivalence (a) $\Leftrightarrow$ (b) is classical; cf. [3], Proposition 50.2.

If (a) and (b) hold, then $X=(I-T)^{k} X \bigoplus \operatorname{ker}(I-T)^{k}$. The subspaces $N:=\operatorname{ker}(I-T)^{k}$ and $M:=(I-T)^{k} X$ are closed and $T$-invariant. Moreover, trivially $(I-T) \mid N$ is nilpotent. Since finite chain length implies that $(I-T) \mid M$ is bijective, (c) follows.

Next suppose $I-T$ is a quasi-Fredholm operator. There are closed $T$ invariant subspaces $M, N$ such that $X=M \bigoplus N,(I-T) \mid N$ is nilpotent, and $(I-T) \mid M$ has closed range containing $\operatorname{ker}\left(((I-T) \mid M)^{n}\right)$ for all $n \in \mathbf{N}$. By Proposition $4 a(I-T) \leq k$, hence $N \subseteq \operatorname{ker}(I-T)^{k}$ and $(I-T)^{k} X=$ $(I-T)^{k} M$. Moreover, $a(I-T)^{k} \leq 1$ and this readily entails that $(I-T)^{k} X \cap$ $\operatorname{ker}(I-T)^{k}=\{0\}$, i.e. $(I-T)^{k} M \cap \operatorname{ker}(I-T)^{k}=\{0\}$. By [10], Lemme I.2.1, $\operatorname{ker}\left((I-T)^{n} \mid M\right) \subseteq(I-T)^{k} M$ for any $n \in \mathbf{N}$, and from this we conclude that $\operatorname{ker}\left((I-T)^{k} \mid M\right)=\{0\}$. It follows that $\operatorname{ker}(I-T)^{k} \subseteq N$. Hence $N=\operatorname{ker}(I-T)^{k}$ and $X=M \bigoplus \operatorname{ker}(I-T)^{k}$. Since $(I-T)^{k} X=(I-T)^{k} M$ is closed, this shows that (d) holds.

Suppose (d) holds. Thus there is a closed $T$-invariant subspace $Z \subseteq X$ for which $X=Z \bigoplus \operatorname{ker}(I-T)^{k}$. Let $W:=(I-T)^{k} \mid Z$. Evidently $(I-T)^{k} X=$ $(I-T)^{k} Z=W Z \subseteq Z$, and $W Z$ is closed. Moreover, $W$ is injective, so there is a constant $c \in \mathbf{R}_{+}$for which $\|W z\| \geq c\|z\|$ for all $z \in Z$. In particular $\left\|(I-T)^{k} M_{p}(T) z\right\| \geq c\left\|M_{p}(T) z\right\|$ for all $z \in Z$ and $p \in \mathbf{N}$. The condition $(E-k)$ says that $\left\|(I-T)^{k} M_{p}(T)\right\| \rightarrow 0$ as $p \rightarrow \infty$, and hence $\left\|M_{p}(T \mid Z)\right\| \rightarrow 0$ as $p \rightarrow \infty$. It follows that $I-M_{p}(T \mid Z)$ is invertible for all sufficiently large $p \in \mathbf{N}$, hence that $1 \notin \sigma\left(M_{p}(T \mid Z)\right)=M_{p}(\sigma(T \mid Z))$. From the form of $M_{p}(z)$ we see that $1 \notin \sigma(T \mid Z)$, i.e. that $(I-T) \mid Z$ is invertible, Hence $W=(I-T)^{k} \mid Z$ is invertible. It follows that $W Z=Z=(I-T)^{k} X$ and since this shows that $(I-T)^{k} X+\operatorname{ker}(I-T)^{k}=(I-T)^{k} X \bigoplus \operatorname{ker}(I-T)^{k}=Z \bigoplus \operatorname{ker}(I-T)^{k}=X$, it follows that (e) holds.

If (e) holds, then by [6], Lemma $1,(I-T)^{k} X$ is closed, so (g) holds.

Clearly (g) implies (h).

Suppose (h) holds. We want to show (i). Let $Y:=(I-T)^{m} X$, where $m \geq k$ is chosen so that $Y$ is closed. Since $(I-T)^{m}: X \rightarrow Y$ is open, there is a constant $c \in \mathbf{R}_{+}$so that for any $y \in Y$ we may pick $x \in X$ so that $(I-T)^{m} x=y$ and $\|x\| \leq c\|y\|$. For any $y \in Y$ and any $n \in \mathbf{N}$ we then obtain $M_{n}(T) y=M_{n}(T)(I-T)^{k} x$, hence $\left\|M_{n}(T) y\right\| \leq\left\|M_{n}(T)(I-T)^{m}\right\|\|x\| \leq$ $c\left\|M_{n}(T)(I-T)^{m}\right\|\|y\|$ from which it follows that $M_{n}(T \mid Y) \rightarrow 0$ as $n \rightarrow \infty$. Arguing exactly as in the implication $(\mathrm{d}) \Rightarrow(\mathrm{e})$ above we conclude that $(I-T) \mid Y$ is invertible, i.e. that $(I-T) Y=Y$. This means that $(I-T)^{m} X=(I-T)^{m+1} X$, i.e. that $I-T$ has finite descent. 
Next assume (i). Since, by Proposition 4, $a(I-T) \leq k$, [3], 38.3, implies that (a) holds.

Finally, it is clear that (a) implies (f) and that (f) implies (g).

Remark. The above Theorem 6 is a partial generalization of [8], Proposition 3.7. In [8], Propositions 3.6, some equivalent conditions on a closed quasiFredholm operator defined on a Hilbert space are listed. These equivalences do not survive as stated, even for contractions which of course satisfy $(E-1)$. For instance, the condition that 1 be a boundary point of $\sigma(T)$ is strictly weaker than Theorem 6 conditions (a) and (b), as the example of the multiplication operator $M_{t}: f(t) \rightarrow t f(t): C[0,1] \rightarrow C[0,1]$ shows. Also, the condition that 1 be an isolated point of the spectrum $\sigma(T)$ is not sufficient for $T$, under the assumption of $(E-1)$, to have a pole at 1 . In fact, if $V$ is the classical quasi-nilpotent Volterra operator on $L^{2}[0,1]$, then $(I+V)^{-1}$ is a contraction, and 1 is an essential singularity of the resolvent.

The next observation is an analogue of [9], Corollaire 2.

Corollary 7. If $T \in L(X)$ has a spectrum $\sigma(T)=\{1\}$, then the following are equivalent.

(a) $I-T$ is nilpotent of order at most $k$.

(b) $T$ satisfies $(E-k)$ and $(I-T)^{m} X$ is closed for some $m \geq k$.

Proof. If (b) holds, then by Theorem $6 X=(I-T)^{k} X \bigoplus \operatorname{ker}(I-T)^{k}$. Since $\sigma(T)=\{1\}$, it follows that $(I-T)^{k} X=\{0\}$.

Recall that an operator $S$ is Riesz-Schauder if it is a Fredholm operator of finite chain length.

Corollary 8. If $T \in L(X)$ has property $(E-k)$, then the following conditions are equivalent.

(a) $I-T$ is a semi-Fredholm operator.

(b) $I-T$ is a Riesz-Schauder operator.

Proof. The implication (b) $\Rightarrow(a)$ is trivial, so we suppose that (a) holds. By the Kato decomposition [4], Theorem 4, $I-T$ is quasi-Fredholm. By Theorem 6 it follows that $I-T$ has finite chain length. But then, by [3], 38.6, $I-T$ is a Fredholm, and hence a Riesz-Schauder, operator.

\section{A UNIFORM ERGODIC THEOREM AND SOME CONSEQUENCES}

The uniform ergodic theorem is about the asymptotic behavior of the sequence $M_{n}(T)$, as $n \rightarrow \infty$. It says that for an operator $T \in L(X)$ for which $\left\|T^{n} / n\right\| \rightarrow 0$ as $n \rightarrow \infty$ the sequence $M_{n}(T)$ is convergent (in operator norm) if and only if $X=(I-T) X \bigoplus \operatorname{ker}(I-T)$ [2], [7]. If $M_{n}(T)$ is convergent in operator norm, we say that $T$ is uniformly ergodic. It is straightforward to see that if $T$ is uniformly ergodic, then $T$ satisfies condition $(E-1)$. In the language that we have introduced here the uniform ergodic theorem may be rephrased as follows: an operator $T \in L(X)$ which satisfies condition $(E-1)$ is uniformly ergodic if and only if $I-T$ has chain length at most 1 . By means of Theorem 6 we shall now state and prove a generalization of this characterization. 
Theorem 9. Suppose $k \in \mathbf{N}$. If $T \in L(X)$ satisfies condition $(E-k)$, then the following conditions are equivalent.

(a) $T$ is uniformly ergodic.

(b) $X=(I-T) X \bigoplus \operatorname{ker}(I-T)$.

(c) $I-T$ has descent and $\operatorname{ker}(I-T)$ has a $T$-invariant complement.

(d) $I-T$ has descent at most 1 .

Proof. Suppose (a) holds. As remarked above, then $T$ satisfies $(E-1)$, so by [7], (b) holds.

Conversely, if (b) holds, then $(I-T)^{k}(I-T) X=(I-T)^{k+1} X=(I-T) X$, so $(I-T)^{k}$ is a bijection of $(I-T) X$ onto itself. The condition $(E-k)$ then clearly implies that $M_{n}(T) \mid(I-T) X \rightarrow 0$ and since $M_{n}(T)|\operatorname{ker}(I-T)=I| \operatorname{ker}(I-T)$, it follows that $T$ is uniformly ergodic.

(b) $\Rightarrow$ (c) is clear and the implication (c) $\Rightarrow(d)$ is part of Proposition 1 .

Finally, if (d) holds, then, by Theorem 6,I $-T$ has finite chain length, and, by [3], 38.3, (d) then shows that this chain length is 0 or 1 . Thus (b) holds.

The above evidently applies to the meromorphic operators, where an operator $T \in L(X)$ is called meromorphic if all nonzero points of its spectrum $\sigma(T)$ are poles of the resolvent function $(T-\lambda)^{-1}: \mathbf{C} \backslash \sigma(T) \rightarrow L(X)$. This class contains all Riesz operators, in particular all compact operators. It also contains all algebraic operators, in particular all projections. If we specialize to meromorphic multipliers on commutative semisimple Banach algebras, then we may characterize uniform ergodicity in quite appealing terms. To specify, assume that $A$ is a commutative semisimple Banach algebra. Recall that a continuous linear operator $T \in L(A)$ is called a multiplier if $a T(b)=T(a) b$ for all $a, b \in A$. In [1], based on [6], Theorem 10, it is shown that a point $\lambda \in \sigma(T)$ is isolated if and only if $\lambda$ is a simple pole of the resolvent $(T-\mu)^{-1}$ and from this it follows that a multiplier $T$ is meromorphic if and only if $\sigma(T)$ is finite or countable and with at most 0 as a cluster point. We have the following result.

Proposition 10. Suppose $A$ is a commutative semisimple Banach algebra and that $T \in L(A)$ is a meromorphic multiplier. Then $T$ is uniformly ergodic if and only if $\sigma(T)$ is a subset of $\mathbf{D}$, the closed unit disc.

Proof. As the containment $\sigma(T) \subseteq \mathbf{D}$ is clearly necessary for uniform ergodicity, we only have to prove the sufficiency of the above condition. By Riesz and Gelfand theory we may split $T=S+W$, where $S$ and $W$ are meromorphic multipliers, with disjoint supports in the maximal ideal space of the multiplier algebra, and with $\sigma(S) \subseteq \mathbf{T} \cup\{0\}$, where $\mathbf{T}$ is the unit circle, and $\sigma(W) \subseteq \mathbf{D} \backslash \mathbf{T}$, the open unit disc. It follows that $W^{n} \rightarrow 0$, from which uniform ergodicity of $W$ is immediate. For $S$, the finiteness of $\sigma(S)$ entails that $S$ is a finite linear combination of idempotent multipliers, the coefficients being the points of $\sigma(S)$. The uniform ergodicity of $S$ is then a simple calculation, and since $S W=0$, the ergodicity of $T$ follows.

\section{REFERENCES}

1. P. Aiena, Some spectral properties of multipliers on semi-prime Banach algebras, Quaestiones Math. (to appear).

2. N. Dunford, Spectral theorem I, Convergence to projections, Trans. Amer. Math. Soc. 54 (1943), 185-217. 
3. H. G. Heuser, Functional analysis, Wiley, Chichester, 1982.

4. T. Kato, Perturbation theory for nullity, deficiency and other quantities of linear operators, J. Analyse Math. 6 (1958), 261-322.

5. J. P. Labrousse, Les opérateurs quasi-Fredholm: une généralisation des opérateurs semiFredholm, Rend. Circ. Mat. Palermo (2) 29 (1980), 161-258.

6. K. B. Laursen and M. Mbekhta, Closed range multipliers and generalized inverses, Studia Math. 107 (1993), 127-135.

7. M. Lin, On the uniform ergodic theorem, Proc. Amer. Math. Soc. 43 (1974), 337-340.

8. M. Mbekhta, Ascente, descente et spectre essentiel quasi-Fredholm, Pub. IRMA, Lille (21), no VI, 1990.

9. M. Mbekhta and J. Zemanek, Sur le théorème ergodique uniforme et le spectre, C. R. Acad. Sci. Paris Sér. I Math. 317 (1993), 1155-1158.

10. A. Ouahab, Contributions à la théorie spectrale généralisée dans les espaces de Banach, Thèse Université des Sciences et Techniques de Lille, 1991.

Department of Mathematics, University of Copenhagen, 2100 Copenhagen, Denmark E-mail address: laursen@math.ku.dk

Department of Mathematics, Université de Lille I, 59655 Villeneuve d'Asque Cedex, FRANCE

E-mail address: mbekhta@gat.univ-lille1.fr 